Revue internationale de pédagogie de

l'enseignement supérieur

$37(1) \mid 2021$

Numéro spécial - hiver 2021

\title{
Télécollaborations (inter)culturelles sur Facebook
}

\section{Elsa Chachkine}

\section{(2) OpenEdition}

\section{Journals}

Electronic version

URL: http://journals.openedition.org/ripes/2976

DOI: 10.4000/ripes.2976

ISSN: 2076-8427

\section{Publisher}

Association internationale de pédagogie universitaire

\section{Electronic reference}

Elsa Chachkine, "Télécollaborations (inter)culturelles sur Facebook", Revue internationale de pédagogie de l'enseignement supérieur [Online], 37(1) | 2021, Online since 14 February 2021, connection on 16 February 2021. URL: http://journals.openedition.org/ripes/2976 ; DOI: https://doi.org/10.4000/ripes 2976

This text was automatically generated on 16 February 2021.

Article L.111-1 du Code de la propriété intellectuelle. 


\title{
Télécollaborations (inter)culturelles sur Facebook
}

\author{
Elsa Chachkine
}

\section{Introduction}

1 La télécollaboration est fréquemment désignée comme favorable pour le développement de compétences interculturelles, parce qu'elle permet de mettre en relation des apprenants distants, qu'elle peut être une alternative à la mobilité pour les adultes en formation - qui travaillent, ont souvent une famille tout en étudiant et ne peuvent s'absenter de leur foyer ou lieu de travail durablement. Cependant, il ne suffit pas de mettre en relation des alloglottes pour développer les compétences interculturelles (O'Dowd, 2006). La conception des tâches demandées joue un rôle central, en favorisant ou non la rencontre interculturelle. De même, le niveau de compétence socioculturelle des apprenants a une influence sur la réussite des échanges (O'Dowd et Ritter, 2006), sur leur engagement dans le projet, sur la rencontre avec l'autre et leurs prises de risque (Audras et Chanier, 2007).

2 Cet article porte sur un projet de télécollaboration entre 17 étudiants du Cnam (France) et 17 étudiants de l'université d'Oulianovsk (Russie). Côté Cnam, le projet de télécollaboration est intégré à un dispositif d'apprentissage du russe en autodirection, qui comprend deux types de télécollaboration, des télétandems et des échanges de productions culturelles sur une page Facebook. C'est ce deuxième type d'échange, qui vise à développer chez les étudiants des savoirs et des savoir-faire socioculturels pour la communication interculturelle, qui est à l'étude dans cet article. Les participants au projet, au Cnam, sont des professionnels qui viennent se former dans le cadre de la formation continue et qui doivent valider un cours de langue, au choix, dans leur parcours de licence ou master ainsi que des apprentis-ingénieur en alternance qui doivent valider une seconde langue étrangère. Côté russe, les étudiants sont des spécialistes de langues (anglais et français), qui se professionnalisent dans le domaine de la communication internationale. 
3 Dans notre contexte, quelle pratique pédagogique innovante est susceptible de favoriser le développement de savoirs et de savoir-faire pour la communication interculturelle? Par «innovant», nous entendons un changement intentionnel, volontaire, délibéré (Cros, 2014, p.172). Parmi les innovations pédagogiques universitaires du moment, Fabre (2015) identifie deux grandes orientations :

4 - L'individualisation : l'étudiant pilote en partie ou totalement sa formation, l'usage du portfolio professionnel et personnel, l'accompagnement personnalisé en sont des illustrations;

5 - la connectivité, qui vise à briser la relation traditionnelle de maitre-élève. L'étudiant est invité à se connecter avec ses pairs pour progresser dans ses apprentissages, créer un réseau de savoirs et un réseau d'apprenants. L'enseignant, lui, est un créateur et gestionnaire de ressources, un animateur que l'on peut solliciter. Cette orientation vise à se dégager du processus " enseigner » pour aller vers le processus « apprendre ».

6 Le projet de télécollaboration à l'étude intègre ces deux grandes orientations pédagogiques - l'individualisation et la connectivité entre pairs. Outre, le développement de compétences langagières, le développement d'un système de références culturelles de base permettant l'interprétation de faits culturels pour la communication interculturelle est recherché. De même, la possibilité de faire vivre aux étudiants des expériences interculturelles et de s'approprier une démarche d'analyse de faits culturels, sachant que, dans notre contexte, les participants du Cnam débutent en russe et ont peu d'expériences interculturelles.

7 Dans la suite de l'article, nous cherchons à comprendre le type de savoirs développés, les savoir-faire mis en œuvre pour la communication interculturelle et esquissons une évaluation du projet, en tentant de répondre à une autre finalité de l'innovation pédagogique, l'efficacité (Tricot, 2017).

\section{Cadre Conceptuel}

8 La télécollaboration inclut des pratiques pédagogiques vastes et une grande diversité d'approches de la recherche (Lewis et al., 2016). Le terme de "télécollaboration » recouvre trois types de télécollaboration identifiés par Dooly et O'Dowd (2012), les télécollaborations entre apprenants relevant de la même institution (premier type), les télécollaborations entre groupes de deux institutions différentes de pays différents (deuxième type), et les télécollaborations où les apprenants d'une institution sont amenés à interagir avec des internautes sur la toile (troisième type, ou télécollaboration 2.0). Nous nous inscrivons dans le deuxième type.

9 La télécollaboration dont il est question dans cet article s'ancre dans la théorie socioculturelle (SCT), une approche de l'apprentissage et du développement mental qui trouve son origine dans les écrits du psychologue russe L.S. Vygotski (Vygotski, 1934/1997) et de ses collègues. Selon cette théorie, les situations d'apprentissage formel anticipent et provoquent le développement et permettent le développement de " concepts scientifiques »; la médiation éducative ainsi que les interactions sociales sont fondamentales pour le développement humain. Cette théorie a orienté la conception du projet de télécollaboration dont il est question dans cet article.

10 La dimension (inter)culturelle est récurrente dans les recherches sur la télécollaboration (Lewis et O'Dowd, 2016). Le modèle de la compétence communicative 
interculturelle le plus souvent mobilisé est celui de Byram (1997). Pour cette recherche, nous adoptons la typologie de compétences socioculturelles pour la communication interculturelle élaborée par Anquetil (2006), qui prend appui sur la typologie élaborée par Byram (1997) et Byram, Zarate et Neuner (1997) mais, à la différence des précédents qui font la part belle aux compétences attitudinales, ce modèle réinsère des compétences comportementales, entendues comme "des capacités à jouer un rôle fonctionnel dans la communication interculturelle, dans un ensemble de compétences socioculturelles plus vastes" (Anquetil, 2006, p. 98) qui inclut des savoirs, des savoirfaire, des compétences méta-culturelles et des compétences d'apprentissage et de méthodologie de l'approche interculturelle. A l'instar d'Anquetil (2006), nous emploierons par commodité le terme de «compétences interculturelles» mais précisons que nous entendons par là « un ensemble de compétences socio-culturelles dont la maitrise favorise la communication» (p. 84). Le terme de "compétence interculturelle» peut, en effet, tendre à réduire le champ des compétences interculturelles uniquement à des compétences transversales de type attitudinales.

11 La catégorie "savoirs" de cette typologie réunit un ensemble de références sur la culture partagée et sur la relation interculturelle. Deux approches complémentaires du concept de culture sont alors utilisées : " une approche de type structuraliste par trait culturel constitutif, et une approche relationnelle (avec les diverses sous-cultures sociales, avec les cultures voisines) et dynamique par l'étude de l'évolution culturelle » (Anquetil, 2006, p. 103). Précisons enfin que le concept de «culture partagée », selon une perspective anthropologique, signifie la culture partagée que des individus de différentes classes sociales ont en commun. Il peut s'agir de la culture scolaire, de certaines chansons, certains personnages, des représentants du monde du sport, etc. (Galisson, 2000).

12 Bien qu'il ne soit pas aisé de parler de savoirs culturels tant le «corps de connaissances» qui peut être envisagé est vaste et presque indéfinissable (Byram, 2012), en nous appuyant sur deux typologies de savoirs culturels, celle d'Anquetil (2006) et celle proposée par l'Unesco, définie comme un «ensemble des traits distinctifs, spirituels, matériels, intellectuels et psychologiques, d'une société ou d'un groupe social et englobe la totalité des manières d'être existant au sein d'une société; elle comprend, au minimum, l'art et la littérature, les modes de vie, les manières de vivre ensemble, les systèmes de valeurs, les traditions et les croyances » (Unesco, 2013, p. 11), nous avons adopté la typologie qui suit. Des savoirs sur les arts, la littérature, l'économie, la politique; la culture partagée (films, chansons cultes, folklore, personnalité connue); le patrimoine, le tourisme; la vie quotidienne et les usages sociaux des produits culturels (coutumes, habitudes, habits, nourriture, loisirs); les conventions sociales et discursives (scripts comportementaux et verbaux, manière de vivre ensemble), les cultures existentielles (mentalités, valeurs).

13 Les savoir-faire comprennent trois opérations majeures de la communication interculturelle: interpréter, interagir et faire œuvre de médiation (Anquetil, 2006, p. 104). A cela s'ajoutent des compétences méthodologiques pour la compétence interculturelle, qui comprend une méthodologie d'apprentissage (expérimentation de stratégie, d'analyse, etc.), des compétences relationnelles (pratique de gestion du contact, multiplier les occasions de contacts, les thésauriser, etc.) et des compétences méta-culturelles, qui incluent des procédures mises en œuvre avant ou pendant la 
communication interculturelle, faisant appel à une activité réfléchie sur les processus culturels en jeu (Anquetil, 2006, p. 320-321).

14 Nous présentons dans la suite de cet article le projet d'échanges de productions culturelles et nous analysons ensuite l'approche de la culture privilégiée dans les publications des étudiants, les compétences socioculturelles pour la communication interculturelle mises en œuvre dans les échanges pour ensuite tenter d'évaluer les apprentissages permis.

\section{Echanges de productions culturelles sur une page Facebook}

15 Le projet d'échanges de productions culturelles sur une page du réseau social Facebook est intégré à un dispositif plus large côté Cnam. Ce dispositif comprend :

- 20 heures d'apprentissage en autodirection avec des ressources pédagogiques (manuels, cours en ligne, carnet de bord, ressources internet);

17 - des télétandems (au moins cinq séances de télétandems de 40-45 minutes doivent être réalisés);

18 - des échanges de productions culturelles, avec tout le groupe, sur une page Facebook;

19 - 2 ateliers collectifs en début et fin de formation pour présenter le dispositif, les partenaires russes pour les télécollaborations, réfléchir à l'apprentissage en autodirection, en télétandem, aux productions culturelles échangées, etc.

20 - 2 entretiens individuels de conseil de 45 minutes avec une enseignante-conseillère pour soutenir l'apprentissage en autodirection, en télétandem et les échanges en grand groupe sur la page Facebook.

Figure 1. Présentation du dispositif de formation et de la façon dont le projet d'échange de productions culturelles s'insère dans le dispositif

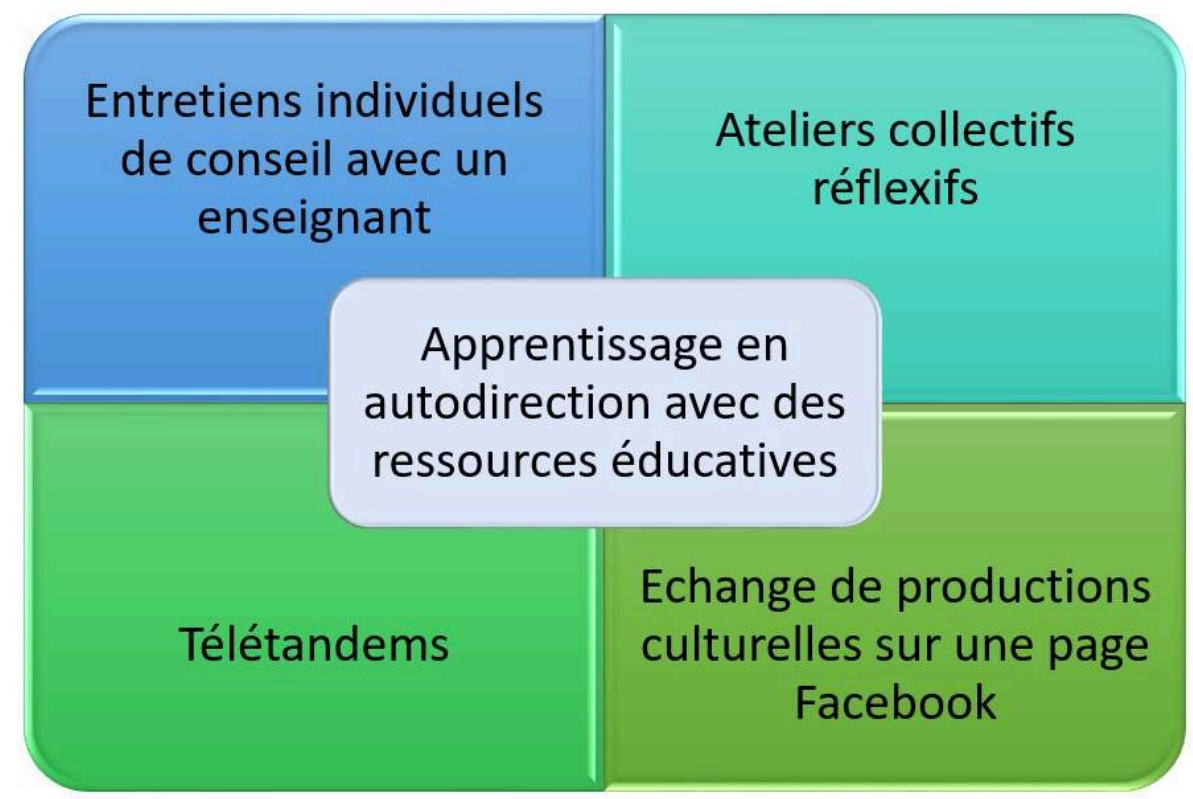


21 S'agissant du projet d'échanges de productions culturelles sur une page Facebook, réservée à l'ensemble des étudiants du Cnam et de l'université d'Oulianovsk, il se présente de la façon suivante : les étudiants (en France et en Russie) postent à tour de rôle une production culturelle (un film, un texte, une vidéo, une photo, une chanson, etc.) de leur pays qu'ils jugent important de faire connaitre et justifient leur choix. Le choix de la publication est fondé sur le fait que c'est un élément de la culture partagée par une majorité de Russes ou de Français ou que c'est un élément de la culture apprécié personnellement par l'étudiant. Dans les deux cas, une explication est attendue. Un calendrier des publications est donné aux étudiants, alternativement un étudiant en Russie, un étudiant en France.

La liberté de choix d'une production culturelle à publier est dans l'esprit du dispositif, qui vise aussi bien l'apprentissage de la langue que le développement de l'autonomie et permet de faire un choix d'un fait culturel qui invite à porter un regard réflexif sur son identité personnelle, professionnelle, etc. au sein de la culture d'origine. Cela implique également de faire le choix de quel type de savoirs privilégier. Cela peut être l'amorce d'une réflexion non seulement sur la multiplicité des significations de ce que peut être une information culturelle (un mode de vie? une chanson? des valeurs? etc.) mais aussi un début de réflexion, à la lecture des publications des autres étudiants, sur la façon dont "chaque personne possède une construction arbitraire du monde, selon ses propres filtres, sa propre réalité » (Zarate, 1986, p. 24), laquelle n'a rien d'universel.

23 Les publications sont marquées par les étudiants d'une des six émoticônes proposées sur Facebook, le plus souvent, dans notre contexte, un pouce levé et parfois un «j'adore». Les publications donnent lieu à des commentaires pour donner une appréciation, demander des compléments d'informations ou exprimer des analogies ou des dissemblances. Les commentaires sont vivement recommandés par l'enseignanteconseillère lors des ateliers et lors des entretiens individuels avec les étudiants du Cnam.

24 Les enseignants russes et français qui encadrent le projet sont présents sur la page, «aiment» toutes les publications postées mais n'interviennent pas dans les commentaires. En revanche, côté Cnam, lors des entretiens de conseil et des ateliers collectifs, l'enseignante-conseillère (nous-même) aide à décrypter certaines publications, invite à commenter en cherchant davantage d'information, à comparer.

25 Au-delà des échanges qu'il permet, cet espace a vocation à fédérer l'ensemble des étudiants, à leur permettre d'élargir leur réseau, de façon à ce que les échanges avec des locuteurs natifs ne se restreignent pas au partenaire de télétandem (Chachkine, 2019).

26 S'agissant du choix de l'outil, Facebook a été choisi pour plusieurs raisons. Nous ne disposons pas au Cnam d'un outil de publication qu'il serait possible de partager avec des personnes extérieures à l'établissement. Facebook offre un large éventail de moyens technologiques (blog, clavardage, visioconférence) permettant de soutenir une grande variété de communication assistée par ordinateur (Blattner et Lomicka, 2012). Le mur de Facebook, qui s'apparente à un blog, favorise le partage d'images, de textes, de vidéos, de liens; les émoticônes peuvent conférer à l'espace une impression de "présence", de même que la fonction "commentaire». Cette fonction " commentaire " permet de comparer, d'apporter des précisions, de développer des liens amicaux ou encore de développer son réseau en devenant « ami » des membres du groupe. Lomicka et Lord (2016) notent que l'utilisation des SNS en classe de langue 
peuvent avoir un effet motivationnel sur les étudiants. Il est vrai, cependant, que si les réseaux sociaux sont largement utilisés par les étudiants pour leur usage personnel, l'usage à des fins d'apprentissage institutionnel peut brouiller les frontières entre le personnel et l'institutionnel et n'est pas toujours bien perçu (Muller, 2015).

\section{Méthodologies} sur la page Facebook, ils sont très nombreux à souhaiter des échanges chaleureux et devenir amis. De part et d'autre, les mobiles utilitaires sont plus rarement mentionnés. Pour les étudiants du Cnam, la participation au projet est obligatoire s'ils veulent valider leur UE. Pour les étudiants d'Oulianovsk, la participation au projet est un bonus, la validation n'est pas obligatoire.

\subsection{Données recueillies et méthodes d'analyse}

Deux types de données ont été recueillies: des données naturelles produites dans le cadre du cours et des données suscitées pour cette recherche. Pour les premières, ont été analysés : 

répondants en France et en Russie. Nous avons procédé à un déchiffrement structurel des réponses et tenté autant que possible de faire table rase d'a priori personnel. Nous avons ensuite réalisé des recoupements entre différentes réponses et nous avons fait émerger des transversalités thématiques. Pour les étudiants du Cnam, nous avons, en plus, comparé le questionnaire d'autoévaluation en début et fin de formation; il sonde les connaissances et compétences linguistiques et culturelles des étudiants ainsi qu'une rubrique sur les compétence interculturelles - restreintes aux politesses ou au degré de conscience que les valeurs peuvent être différentes d'une culture à l'autre. Les carnets de bord des étudiants du Cnam ont été très peu utilisés, les réflexions qui y sont consignées étant quasiment absentes et les apprentissages réalisés très peu notés.

\section{Analyse des productions culturelles et commentaires échangés tout au long du semestre}

\subsection{Analyse des publications échangées, thématiques et positionnement énonciatif}

\section{Domaine de savoirs privilégiés par les étudiants}

Les publications des étudiants du Cnam sont principalement relatives à la vie quotidienne et aux usages sociaux des produits culturels (5). Ces publications portent sur l'apéritif, la quiche lorraine, le champagne, la chocolatine (ou pain au chocolat) ou 
encore sur le festival interceltique de Lorient. Le second type de publication est relatif au tourisme, au patrimoine. Un autre type de publication privilégié (4) concerne un domaine qui comprend les arts, les sciences, l'histoire. Dans ce domaine, les sujets abordés sont l'histoire de France, l'histoire du français, le système politique français ou encore le Cnam et ses missions. Trois publications concernent la culture partagée, des références culturelles connues par un très grand nombre d'habitants en France, comme Gainsbourg ou Aznavour; une troisième publication de cet ensemble porte sur le luxe et Paris. Cette dernière publication relève aussi bien d'une culture partagée que d'une identité attribuée, l'étudiant chercherait alors à « jouer » au Français, selon une sorte de mise en scène identitaire (Dervin, 2011), à moins que l'intention de l'étudiant soit de mettre en œuvre une technique de "séduction culturelle ", pour rassurer l'autre en le confortant sur une partie de ses représentations des caractéristiques "nationales" habituellement attribuée aux Français, et ainsi engager le dialogue (Anquetil, 2006). Une seule publication porte sur les conventions sociales, les manières à table, avec un support vidéo dans lequel sont recensés 40 « bonnes manières ", que l'étudiants précise ne pas respecter, ni lui ni son entourage. Enfin, une seule publication porte sur les pensées et les valeurs et traite de la différence de vie dans une très grande ville (Paris, qui est le lien d'étude de l'étudiant) et la campagne (d'où l'étudiant vient).

Les thématiques choisies par les étudiants de l'université d'Oulianovsk, ville qui se trouve au bord de la Volga à l'est du continent européen à $700 \mathrm{~km}$ au sud-est de Moscou, portent sur des informations touristiques (5), des informations sur les arts (4) et des informations sur des personnalités ou chanteurs connus par une très grande majorité d'habitants en Russie, que nous nommons "culture partagée " (4). Les informations de type touristiques portent sur la ville d'Oulianovsk ou des sites environnants (comme l'île de Paltsinski, formée en 1950, réservoir ornithologique dans la ville); les autres sites touristiques présentés sont en Sibérie orientale, la péninsule Kamchatka avec ses 300 volcans inscrits au patrimoine de l'UNESCO et le lac Baïkal. Les publications sur les arts concernent le compositeur Piotr Ilitch Tchaïkovski, le ballet russe, un peintre marin du $19^{\text {ème }}$ siècle Ivan Aïvazovski ou encore un musée de la photographie d'Oulianovsk. Les célébrités présentées sont des sportifs soviétiques (comme par exemple Boris Mikhaillov, joueur de hockey et entraîneur sur lequel un blockbuster russe «Legend N 17 » est récemment sorti); un chanteur de variété russoukrainien d'origine lettone, Vitas, qui a terminé sa carrière en Chine. Dans cette catégorie, une autre publication porte sur des symboles russes : l'intention de l'étudiant est de montrer autre chose que la place rouge, le froid et les ours et propose de présenter d'autres symboles. Dans le domaine des savoirs sur les conventions sociales et discursives et les manières de vivre ensemble, une publication est proposée sur le rapport aux animaux domestiques dans une ville de bord de mer (Zelenogradsk, dans une enclave territoriale entre la Pologne et la Lituanie, où les chats sont choyés et adulés). Enfin, une publication porte sur les mentalités, les valeurs, avec une publication sur les superstitions en Russie.

La variété des sujets abordés, côté Cnam et Oulianovsk, est susceptible de faire prendre conscience de combien le champ des savoirs sur les cultures est vaste et combien ces savoirs peuvent être abordés selon des angles d'approche variés (art, vie quotidienne, culture partagée, manière de vivre, croyances). Dans les choix réalisés entre les étudiants du Cnam et d'Oulianovsk, on ne relève pas de différences frappantes dans le type de production culturelle choisi. Comme les publications sont alternativement publiées par un étudiant du Cnam et un étudiant d'Oulianovsk, il est très probable que 
les étudiants se soient ajustés aux publications qui ont précédé et qu'ils aient été influencés dans leurs choix par les publications qui reçoivent le plus d'« aimer » et les plus commentées. On note toutefois que le champ de la vie quotidienne est beaucoup plus investi par les étudiants du Cnam. Il peut s'agir de mettre en avant le savoir-vivre mais aussi d'insuffler un caractère décontracté aux échanges. Côté Oulianovsk, le tourisme local est passablement mis en valeur dans les thématiques mais aussi dans la posture énonciative adoptée: "notre ville", "notre région" s'oppose à la capitale, Moscou, et à Pétersbourg que tout le monde connait. Pour le reste des publications sur cette thématique, c'est le patrimoine géographique qui est à l'honneur, du Kamtchatka, à l'extrême est, à l'enclave de Kaliningrad, à l'extrême ouest, c'est l'image du pays continent qui se dégage prioritairement.

\section{Présentation générale des publications et positionnement énonciatif}

Chaque publication débute par un texte de 200 mots environ suivi de vidéos et ou de photos qui viennent illustrer le texte introductif ou de liens internet qui renvoient vers des sites qui permettent d'approfondir la thématique abordée. En cela, les applications $\mathrm{du}$ Web 2.0 et les réseaux sociaux, " par leur orientation collective, ancrée dans leurs fonctionnalités spécifiques (par exemple, commenter, relier, cosigner, réviser, remixer, partager, aimer, etc.), non seulement façonne de nouvelles formes d'interactions en ligne, souvent de nature multimodale, mais offrent également des possibilités de nouvelles formes d'activités collectives pour l'apprentissage des langues » (Blin, 2012, p. 79). Les publications sont diversement « aimées » (de 14 à 28 j'aime) et commentées également de façon variables (de 0 à 22 commentaires), les publications en début et milieu de semestre étant beaucoup plus commentées qu'en fin de semestre, ce qui correspond à la période des examens de part et d'autre mais aussi à un possible essoufflement du groupe après 3 mois d'échanges.

Comme cela est demandé dans la consigne, les étudiants sont invités à publier une information culturelle de leur pays d'origine qu'ils apprécient et qu'ils ont envie de faire connaitre à l'ensemble du groupe. Il leur est également demandé d'expliquer leur choix et de préciser si ce qui a été choisi est apprécié en général dans son pays d'origine (est-ce qu'une majorité de Russes ou de francophones apprécient ce que vous avez choisi? Ou est-ce apprécié par une petite minorité de personnes? Et, si oui, laquelle? Des jeunes, des moins jeunes? Des étudiants? etc.). La publication se présente dans la très grande majorité des cas par une marque d'adresse à l'ensemble du groupe "Bonjour à toutes et à tous!", en français et en russe, suivie d'une émoticône souriante, une explication sur la raison du choix qui se limite le plus souvent au fait d'aimer le sujet choisi ou d'avoir vécu dans le lieu choisi ou encore parce que la thématique est en lien avec son lieu d'origine « Aujourd'hui je vais vous parler d'un plat traditionnel de la région d'où je suis né » (ETU Cnam). Suivent des explications extraites de sites, complétées de photos, parfois de vidéos et de liens complémentaires pour approfondir le sujet abordé. Une question termine la publication et invite à comparer «Et vous? Avez-vous déjà goûté à ce plat? Quel est le plat emblématique de votre région? » (ETU Cnam) 
Figure 2. Exemple de publication d'une étudiante d'Oulianovsk.

8 novembre 2019

Bonjour à tous!

J'aime prendre des photos comme vous le savez déjà de notre connaissance, c'est pourquoi je veux vous parler

du musée de la photographie de Simbirsk.

Le Musée d'histoire de la photographie de Simbirsk est situé dans un ancien manoir marchand de la rue

Enguels. A l'entrée du musée se trouve une composition sculpturale, c'est un vieux photographe avec un appareil photo archaïque sur un trépied.

Au début du XXéme siècle, un studio photo est ouvert dans la maison. En charge de tout étaient deux personnes: Kholevine et Nikanorov. Sur la base de leur atelier de photographie on a décidé de créer un musée "photographie de Simbirsk». II a été ouvert en 2004. II y a partout des attributs du début du dernier siècle: meubles anciens, objets et outils du studio photo - vieux appareils photo, trépieds, équipement pour développer des photos. La plupart des expositions du musée appartenaient aux photographes de Simbirsk qui ont vécu il y a cent ans. On dit que de temps en temps des fantômes apparaissent dans ce musée. :

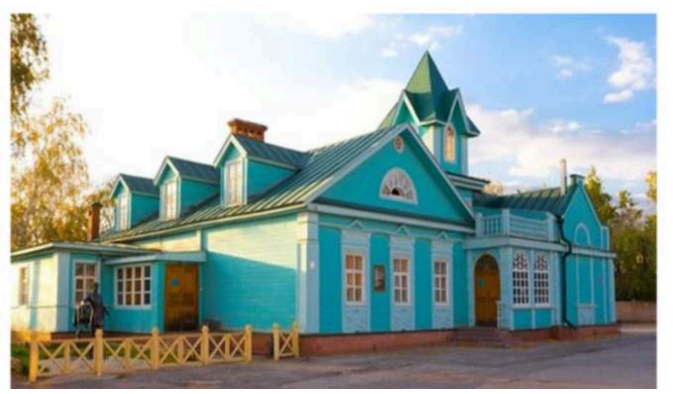

La première phrase est très généralement introduite par un pronom à première personne, « je », qui explique l'origine et le choix de la publication « Je vais vous parler d'un festival qui me tient à cœur, le Festival interceltique de Lorient » (ETU Cnam). Ce script verbal, adopté par les étudiants du Cnam en début de semestre, semble adopté comme modèle par les étudiants d'Oulianovsk pour leurs présentations «J'adore la musique, c'est pourquoi aujourd'hui je vais vous parler d'un compositeur russe de l'époque romantique, à savoir Piotr Ilitch Tchaïkovski » (ETU Oul). Le choix de la thématique et sa provenance posés, les étudiants entament ensuite une description avec des informations assez factuelles, faiblement modalisée, tonalité que l'on peut trouver dans Wikipédia ou dans des dictionnaires des noms propres : « Le lac Baïkal (en russe : ОЗЕРО БАЙКАЛ, Ozero Baïkal) est un lac situé dans le sud de la Sibérie, en Russie orientale. Il constitue la plus grande réserve d'eau douce liquide à la surface de la Terre » (ETU Oul), descriptions auxquelles sont ajoutées, parfois, des émoticônes qui personnalisent un peu la description.

Si les étudiants optent très majoritairement pour cette description factuelle, certains optent pour un «notre pays " ou "notre histoire » ou encore "notre langue » pour s'inclure dans une entité distincte de l'autre entité qu'est la Russie : « Alors laissez-moi, sans plus attendre, vous parler de notre beau pays et de son histoire » (ETU Cnam) ou encore « Grâce à la position géographique de la France en Europe, nous avons la chance de disposer de la Manche, de l'océan Atlantique et de la Méditerranée pour un maximum de diversité. Notre Histoire nous a aussi gâté...» (ETU Cnam). Le possessif " notre ", la tonalité emphatique adoptée avec la locution verbale "nous avons la chance de" ou l'adjectif «beau» qui réfère au pays, la France, la locution prépositionnelle " grâce à » témoignent d'une posture différente et d'un genre textuel de type promotionnel. Chez les étudiants d'Oulianovsk, on note quelques descriptions élogieuses de leur ville ou de leur région dans les commentaires où "notre ville ", 
«notre région » s'oppose aux deux grandes villes européennes russes que sont Moscou et Pétersbourg «Bien sûr, tout le monde connaît Moscou, Saint-Pétersbourg... Mais la Russie ne se limite pas à ces endroits » (ETU Oul).

\subsection{Analyse des commentaires et des traces de savoir-faire socioculturels pour la communication interculturelle}

Nous poursuivons avec l'analyse des échanges écrits dans la fonction "commentaire " des publications. Les publications donnent lieu à l'échange de 280 commentaires, dont la longueur oscille de 0 mots à 140 mots. Des échanges de photos sont également réalisés dans cette fonction commentaire. Ces échanges permettent d'affiner les connaissances introduites dans la publication de départ, sur la vie quotidienne et les usages comparés de produits culturels notamment, comme dans ce commentaire par exemple «Le ballet est vraiment un art très envoûtant (-) Malheureusement, je n'ai jamais regardé le ballet, mais je voudrais (ETU Oul). Si le ballet russe semble très apprécié en Russie et largement regardé, ce commentaire permet de nuancer et de s'apercevoir qu'en Province, l'accessibilité n'est peut-être pas si simple.

Ces commentaires sont aussi l'occasion de mettre en œuvre des savoir-faire liés à trois types d'actions jugées "essentielles pour la communication interculturelle, interprétation, interaction et médiation" (Anquetil, 2006, p. 99), ainsi que des compétences métaculturelles. Nous proposons de relever les savoir-faire les plus fréquemment mis en œuvre.

Une caractéristique frappante des échanges est son caractère laudatif, très amplifié au début des échanges. On note de très nombreux " magnifique " (18 occurrences), «beau» (16), «joli» (9), grandiose (4), «délicieux» (5) et une pléthore de remerciements (84). Cela confère un aspect un peu convenu et policé aux échanges mais la fonction est très certainement de séduire socialement, de créer le contact et d'engager l'échange.

Utilisation de différentes stratégies de séduction sociale pour forcer le contact

La séduction sociale ne s'exprime pas uniquement par des commentaires laudatifs et les nombreux remerciements, mais également par le désir d'apprendre la langue de l'autre, comme ce message d'un étudiant du Cnam rédigé à l'aide d'un traducteur automatique «[...] МЫ НАЧИНАЕМ ИЗУЧАТЬ РУССКИЙ ЯЗЫК 9 ОКТЯБРЯ. Я НЕ МОГУ

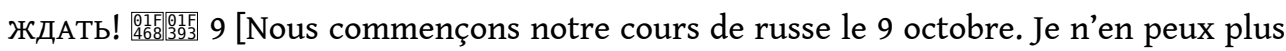

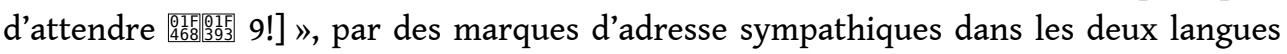
« ПРИВЕТ ВСЕм! Bonjour tout le monde! J'espère que vous allez tous bien! (-) » et les émoticônes qui expriment tantôt des sourire, des allures décontractées, des mains pour aider, etc., rendant ainsi l'espace accueillant et invitant à s'exprimer sans crainte.

Cette séduction s'exprime également par l'expression du désir de venir voir le pays de l'un ou de l'autre. À la suite d'une première publication d'une vidéo sur les différentes régions de France, les étudiants du Cnam expliquent, dans les commentaires, la région d'où ils viennent, vantent les atouts culinaires, esthétiques et le savoir-vivre d'une région et ajoutent à leur commentaire une photo ou une vidéo qui la présente plus en détail. En forçant le trait sur l'attrait de leur région, ils invitent à aimer leur région, à venir la visiter. En retour, les étudiants d'Oulianovsk remercient, promettent une visite 
dans chacune. Ce premier échange invite à aller au-delà des télécollaborations et confère à l'espace un climat sympathique :

«- ETU Cnam 3 : Vive la région Occitanie et surtout le département de l'Aveyron! 3

- ETU Cnam 4 : C'est le département qui comporte le plus grand nombre de "plus beaux villages de France". On y fait du Roquefort, fromage mondialement connu! https:// www.tourisme-aveyron.com/.../decouvrir-aubrac-en...

$\underline{3}$

57 - ETU Cnam 5: Je confirme que l'Aveyron est une formidable région, avec de magnifiques paysages et des villages très pittoresques. Je me souviens d'un weekend passé à Najac, un bel endroit à découvrir...

$\underline{1}$

- ETU Oul : Merci beaucoup pour cette information! Quant à moi, je ne peux pas choisir une région de la France que je préfère parce qu'elles sont différentes, uniques et si belles. Je voudrais visiter toutes les régions:) 3 [...] ».

58 Cet échange qui mobilise des techniques de séductions culturelles pour aménager le premier contact, pour susciter la curiosité et instaurer une convivialité participe non seulement de savoir-faire liés à l'interaction dans un espace multiculturel mais également de la médiation, dans le sens où l'échange prend explicitement en compte la différence et l'intègre dans un jeu social pour médier sa culture. Il s'agit moins de jouer à l'Aveyronnais ou d'une mise en scène identitaire comme le déplore Dervin (2011) que de créer un espace tiers de partage pour présenter, traduire, expliciter sa culture.

Demande d'informations, par la comparaison, pour interpréter plus justement

60 Un autre savoir-faire fréquemment mis en œuvre dans les commentaires est le fait d'aller chercher des informations supplémentaires auprès des étudiants d'Oulianovsk ou du Cnam pour tenter d'interpréter un produit culturel ou une représentation. C'est généralement par la comparaison entre pays que les questions sont posées. En voici un exemple :

61 «- ETU Cnam : Et en Russie, quels sont les événements qui ont le plus marqué l'histoire de votre pays?

- ETU Oul 1: A mon avis, ce sont la Grande révolution socialiste d'Octobre de 1917 qui a marqué l'histoire non seulement de la Russie mais du monde entier et la Grande Guerre Patriotique de 1941-1945. $\underline{3}$

- ETU Oul 2 : [...] Je crois qu'il y a beaucoup d'événements importants pour notre pays. Mais le plus important est considéré, bien sûr, la Grande Guerre Patriotique, ainsi que la Reconstruction des années 1980. »

62 Cet échange permet de prendre conscience que la révolution russe de 1917 (nommée «Grande révolution socialiste d'Octobre de 1917 »), la seconde guerre mondiale (nommée différemment en Russie par «Grande Guerre Patriotique» et de date différente 1941-1945) et la perestroïka sont les trois événements historiques jugés les plus marquants par ces étudiants d'Oulianovsk. Il s'agit-là de chercher à identifier les représentations de la culture étrangère par les locuteurs eux-mêmes de cette culture.

Dans un autre exemple qui porte sur l'apéritif en France, un étudiant va chercher à comprendre si la nature de la boisson varie en fonction des saisons :

64 «- ETU Oul Merci pour votre information qui est très intéressante. Surtout le vidéo qui contient l'information historique m'a paru très curieux. Chaque peuple est caractérisé

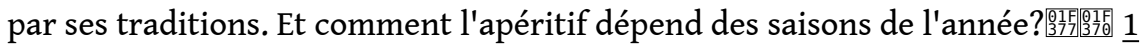


- ETU Cnam里囬 ETU Oul 1! Si tu as des questions sur la vidéo tu peux me les poser, je serais ravi de te répondre.

L'été on aime prendre l'apéro dehors lorsqu'il fait chaud5255 et boire des boissons fraîches . Sinon il n'y a pas vraiment de différences selon les saisons :) »

Cet échange permet à l'étudiant du Cnam de lever quelques implicites sur l'apéritif, le fait que les boissons sont froides et l'accompagnement salé, ce qui rend impossible de rapprocher la pratique de l'apéritif à celle du thé.

Les questions, les comparaisons invitent à davantage expliciter et décomposer les pratiques. Il s'agit d'un autre savoir-faire amplement mis en œuvre dans les commentaires.

Explicitation du cadre de référence implicite dans lequel s'inscrit le produit culturel

a nature de la tâche est justement de présenter, d'expliciter une production culturelle. Mais les commentaires permettent parfois d'approfondir le sujet, lorsque des participants au projet demandent une précision, comparent, reformulent leur compréhension. Cela invite à lever des implicites auxquels les étudiants n'avaient pas pensé, à décomposer les pratiques et éviter de les imposer comme des évidences.

«- ETU Oul: Bonsoir, merci beaucoup pour les informations intéressantes sur les apéritifs en France! Moi, j'aime beaucoup cette tradition! En Russie, l'apéritif en tant que tel est peut-être plus une bouffée amicale, quand il n'y a pas de déjeuner traditionnel [...] C'est juste du vin, de la bière, du jus, des snacks, des sucreries, etc. Mais, comme vous le comprenez, la Russie est pays multinational, et par exemple, certains peuples font l'apéritif avec des sucreries et du thé (avant le déjeuner!) [...].:.: 2

- ETU Cnam: Bonjour Etu Oul! À savoir qu'en France l'apéritif ne se fait pas nécessairement avant tous les repas :) On "prend l'apéritif" en général lorsqu'on invite des amis ou de la famille chez nous ou pour se décontracter après une longue journée de travail.

C'est intéressant de savoir qu'il existe d'autres sortes d'apéritifs en Russie :D $\underline{1}$ ”

Dans cet échange, l'étudiante d'Oulianovsk interprète la pratique de l'apéritif en comparant à d'autres usages ressemblant en Russie, pratiques qui semblent plus s'apparenter à manger un morceau avec des amis et prendre le thé dans le deuxième exemple. L'étudiant du Cnam précise alors qu'il s'agit d'une autre forme d'apéritif (qui n'est ni chaud, ni sucré ni un repas). Cet échange permet de développer des savoirs sur les usages sociaux de l'apéritif en explicitant le cadre de référence implicite (pas avant tous les repas) et de mettre en œuvre le savoir-faire d'interpréter en comparant, en tentant de mettre en évidence des équivalences, tentative de mise en équivalence qui est réajustée par l'étudiant du Cnam.

Espace de connivence culturelle

72 Cet espace est également un lieu où les membres du groupe apprennent à se connaitre, à échanger sur des affinités culturelles. Les passionnés de photographie se partagent des photographies, ceux qui aiment la musique classique échangent sur leur compositeur favori ou pièces favorites, etc., ce qui, à l'intérieur du grand groupe, crée des sous-groupes qui partagent des connivences culturelles:

73 «- Etu Oul 1: Merci Ksenia! J'aime bien la musique classique, surtout les pièces pour piano. 8

- Etu Oul 2: Salut tout le monde! (-) Je voudrais ajouter que les parents de Tchaikovsky n'étaient pas musiciens et d'après sa première formation il était juriste 5 
- Etu Cnam 1: Oui c'est rigolo qu'à cette époque-là beaucoup de musiciens russes célèbres avaient une deuxième profession. Borodine, lui, était chimiste, Cui ingénieur... 1

- Etu Cnam 2: Merci pour cette présentation Ksenia! J'aime également beaucoup Sergueï Rachmaninov, notamment sa deuxième symphonie. Est-il aussi connu que Tchaïkovski en Russie? 5

-Etu Oul 3 Oui Etu Cnam 2, il est connu et aimé! »

L'humour, toutefois, reste confiné au sein de sous-groupe d'un même établissement, ce qui ne manque pas d'être relevé par un étudiant, qui explicite sommairement :

«-Etu Cnam 1: Afin d'attiser les tensions culturelles entre la france et le sud ouest, l'appellation chocolatine est toléré. Cependant, à Paris on dit pain au chocolat! Paris c'est la France donc si vous visitez notre beau pays dites pain au chocolat c'est plus simple. Les gens du sud ouest ne seront pas vexé car ils ne voyage pas. 9 Haha

- Etu Cnam 2 : Ah ah, ça c'est très French private joke! La démarcation chocolatine/pain au chocolat est surtout sud/nord $\underline{1}$ "

Peu de mise en cuvre de compétences méta-culturelles ou de changement de représentations

Beaucoup plus rarement, les échanges dans les commentaires donnent lieu à des changements de représentations, à une activité réfléchie sur les processus culturels en jeu, comme la reconnaissance d'une forme d'ethnocentrisme ou le fait de porter un regard réflexif sur son/ ses identité/s. Ils ne sont toutefois pas absents, en voici quelques illustrations :

78 «Etu Cnam : Puisqu'on parle d'histoire, je suis toujours surpris à quel point on sousestime, en France, l'importance de la Russie dans la défaite allemande à la fin de la seconde guerre mondiale. Pour beaucoup de nos compatriotes, ce sont les Américains (et un peu les Anglais et les résistants français...) qui ont vaincu les nazis... 1 »

Un autre échange permet de lever une représentation erronée sur le climat en Russie, envisagé comme toujours froid. L'échange permet d'ajuster la représentation de l'étudiant du Cnam :

80 «- Etu Cnam 1 : Magnifiques paysages dommage qu'il fasse si froid en Russie 3

- Etu Oul 1: Etu Cnam 1 il ne fait pas toujours froid en Russie. Bon, ce dépend d'endroit. Par exemple, il fais très chaud en été dans notre région ${ }_{0377}^{017} 0^{7} 4$

- Etu Cnam 1: Etu Oul 1, je suis curieux de savoir combien de degrés il fait en été en Russie à l'endroit où vous étudiez? 1

- Etu Oul 1: Etu Cnam 1 la température moyen est à propos de $26{ }^{\circ} \mathrm{C}$, en fait, la température peut atteindre 30 degrés et plus 1

- Etu Cnam 1 : Etu Oul 1 ça va je m'attendais à un peu plus froid quand même 1 »

81 D'autre part, on ne relève aucun incident critique dans les publications et commentaires. Les rares échanges qui auraient pu donner lieu à des dissensions ne sont pas développés, ils semblent contenus, comme dans cet échange qui porte sur l'antisémitisme d'état sous Brejnev :

« - Etu Cnam : Salut Etu Oul! Sujet très intéressant. J'adore les œuvres de Tchaïkovski et notamment son "Concerto in D Major op 35" qui est joué dans un film Français peu connu: Le Concert. Ce film raconte l'histoire de l'Orchestre du Bolchoï dirigé par Filipov, qui avait été interrompu lors d'une représentation où l'orchestre jouait ce concerto justement. En effet, Andreï [Filipov] avait refusé, à l'époque de Brejnev, de se séparer de ses musiciens juifs, ce qui n'avait pas plu au gouvernement... Plus 
d'informations sur ce lien : https://fr.m.wikipedia.org/wiki/Le_Concert_(film) 6 J'adore

- Etu Oul : Etu Cnam Merci! Je regarderai ce film :) 1 »

83 Si les rares occasions de sujets possiblement conflictuels ne sont pas développés dans les commentaires, ils peuvent l'être entre étudiants lors des télétandems (un étudiant du Cnam et un étudiant d'Oulianovsk s'apprennent mutuellement leur langue tout au long d'un semestre). Côté Cnam, certaines publications sont décryptées lors des entretiens individuels de conseil et lors de l'atelier collectif réflexif, qui visent à donner des clés de compréhension de certaines publications et échanges.

\subsection{Evaluation globale de la tâche par l'ensemble des apprenants et autoévaluation des apprentissages}

\subsubsection{Evaluation globale par les questionnaires}

L'évaluation de la tâche par les étudiants d'Oulianovsk (9 répondants) et du Cnam (11 répondants) est très positive. A la question "Comment qualifier les échanges sur Facebook? », en France comme en Russie, les étudiants évaluent la tâche très favorablement «très bien ", « très intéressante ", « utile et conviviale ».

S'agissant des apprentissages réalisés et d'un possible changement dans les représentations sur les uns et les autres, les réponses sont sensiblement différentes. Les étudiants du Cnam, qui débutaient en russe, déclarent avoir développé de nombreuses connaissances sur la culture, «sur le fait que beaucoup d'artiste français sont connus là-bas ", " découvert le mode de vie des russes en partie ", " des endroits, des artistes, des façons de vivre dont j'ignorais totalement l'existence » mais aussi des éléments " sur la manière dont chacun perçoit sa propre culture. Par exemple, j'ai remarqué que les étudiants français voyaient leur culture principalement à travers leur culture culinaire et territoriale, alors que les étudiants russes voyaient la leur plutôt à travers leur art (poésie, art visuel, etc.) et leurs paysages naturels ». Cinq étudiants du Cnam sur 11 déclarent même avoir, suite à cette expérience, modifié leur représentation de la Russie et des Russes: "Très nettement car j'étais encore envahie de représentations anciennes ", "sur la mentalité de la jeune génération, plus moderne que ce que je pensais ", " plus ouverts sur le monde que ce je pensais ", ou ont enrichi leur représentation "Ma vision de la Russie se limitait avant aux éléments d'actualité (politique étrangère notamment). Maintenant j'ai des éléments et opinions directement reçus d'habitants russes ». Parmi les publications qui ont séduit les Etudiants du Cnam, ce sont celles qui portent sur des sites géographiques (Kamchatka, les lacs russes). Cette approche «touristique » se retrouve d'ailleurs dans les rapports de séjour de mobilité d'étudiants (Anquetil, 2006).

Les étudiants d'Oulianovsk, de niveau intermédiaire en français, déclarent également avoir développé des connaissances au sens large « Я УЗНАЛА НОВУЮ ИНФОРМАЦИЮ О КУХНЕ ФРАНЦИИ, О ФЕСТИВАЛЯХ [des informations sur la cuisine, sur les festivals] « О ФРАНЦУЗСКОЙ МОЛОДЕЖИ » [sur les jeunes en France] « ТРАДИЦИИ ФРАНЦИИ, МЕСТА, КОТОРЫЕ МОЖНО ПОСЕТИТЬ » [sur des traditions, des endroits à visiter], «О ЖИЗнИ ФРАНЦИИ » [sur la vie en France] mais aussi la pratique du français et des savoirs en pragmatique socioculturelle sur les scripts verbaux des messages «ТАКЖЕ Я СМОГЛА ПОПРАКТИКОВАТЬ СВОЙ НАВЫК ФРАНЦУЗСКОГО ЯЗЫКА » [j’ai pu également pratiquer 
mon français] ou « ТАКЖЕ УЗНАЛА НЕКОТОРЫЕ ПРАВИЛА НАПИСАНИЯ СООБЩЕНИЙ НА ПРИМЕРЕ ПУБЛИКАЦИЙ [j'ai également appris quelques règles de rédaction de messages en prenant exemple sur les publications]. S'agissant de changements dans leurs représentations, les étudiants ne déclarent aucun changement, ce qui semble assez logique dans la mesure où les étudiants d'Oulianovsk ont un niveau intermédiaire en français et que les publications proposées portent généralement sur des sujets connus. D'autre part, côté russe, l'accompagnement par un enseignant est minimalement proposé, il est probable que cela n'ait pas aidé les étudiants à traduire, interpréter des productions culturelles échangées.

87 A la question « Avez-vous apprécié donner des informations culturelles sur votre pays? Le choix de l'information culturelle à partager vous a-t-il posé problème? Si oui, expliquez», tous déclarent avoir grandement apprécié, avoir pris conscience de combien les cultures sont métissées, mouvantes : «Oui. Chaque pays et peuples ont des histoires qui, pour toutes à un moment donné, se sont entremêlées, mélangées. C'est pour ça que j'ai d'ailleurs préféré orienter ma publication vers l'histoire » ou déclarent avoir été un peu désarçonnés par la multitude des choix possibles «Le choix était tellement vaste que c'était compliqué de choisir » ou encore d'avoir apprécié donner des informations auxquelles les uns et les autres n'auraient pas pensé.

Enfin, à la question « Avez-vous été gêné.e par une publication ou des commentaires? Si oui, laquelle (lesquelles)?», aucune gêne n'est relevée: «НЕТ, ВсЕ СТАРАЛИСь БЫТЬ ВЕЖливыми » [non, tout le monde a fait en sorte d'être poli].

Le caractère écrit, institutionnel, collectif, visible aux deux enseignantes française et russe ont pu favoriser une retenue, ce qui n'est pas le cas des échanges dans le cadre des télétandems ou les échanges, parfois quotidiens via What App mis en place de plein gré par certains tandems. Mais ces données ne sont pas accessibles au chercheur du fait que les télétandems, les échanges informels n'ont pas été enregistrés.

\subsubsection{Autoévaluation des connaissances développées par les étudiants}

90 Une autoévaluation des connaissances des étudiants avant le démarrage de la formation et en fin de formation a été demandée aux étudiants du Cnam. Inclus dans leur carnet de bord, l'autoévaluation porte sur leurs compétences langagières, leur connaissance sur la culture russe et leurs compétences interculturelles et sonde également leur capacité d'autodirection. Adaptée à des apprenants de russe débutants ou faux débutants, l'auto-évaluation comporte une échelle de 0 à 10, de " pas capable du tout » qui s'exprime sur l'échelle par 0 , à « tout à fait capable », qui s'exprime par 10. L'auto-évaluation invite à réfléchir aux connaissances dont les étudiants disposent et peut constituer une aide pour se fixer des objectifs langagiers parmi les items proposés pour le travail en autodirection. A l'issue de la formation, ils réalisent une deuxième auto-évaluation. L'ensemble du dispositif d'apprentissage du russe est concerné par cette auto-évaluation. Nous ne présentons toutefois que le volet culturel et interculturel dans le tableau suivant.

Tableau 1. Volet culturel et interculturel de l'auto-évaluation

1 - Vous avez des connaissances sur la culture russe (donnez un chiffre pour chaque item) 


\begin{tabular}{|l|l|}
\hline & 1.1 - La vie quotidienne des Russes \\
\hline & 1.2 - La vie des étudiants en Russie \\
\hline & 1.3 - Les arts (musique, cinéma, littérature, etc.) \\
\hline & 1.4 - Géographie, reliefs, paysages, climat \\
\hline & 1.5 - Le sport \\
\hline $\begin{array}{l}\text { 2- Vous avez des compétences interculturelles de base (Vous savez, par exemple, que vos propres } \\
\text { règles de politesse peuvent être partagées ou non par les Russes) }\end{array}$ \\
\hline
\end{tabular}

91 Si nous croisons (cf. figure ci-dessous) les connaissances autoévaluées en début de formation (axe des ordonnées) aux apprentissages permis en fin de formation (axe des abscisses), grâce aux échanges de productions culturelles mais aussi aux télétandems et au travail en autodirection, on note que les connaissances de départ en histoire et politique ainsi qu'en géographie, relativement importantes en début de formation, sont faiblement développées tout au long du semestre. Par contre, les étudiants qui ne possédaient quasiment aucune connaissance sur la vie des étudiants en Russie et sur le quotidien des Russes en début de formation ont développé le plus de connaissances sur ces deux thématiques. Les arts et le sport arrivent également en bonne place: moyennement connus en début de formation, ils sont notablement développés en fin de formation. Nous précisons que cette autoévaluation porte sur des connaissances et compétences développées pour l'ensemble du dispositif d'apprentissage et non spécifiquement sur le projet d'échanges de production culturelle. Nous faisons toutefois l'hypothèse que la tâche d'échange de productions culturelles contribue à la constitution d'un socle de connaissances, sans savoir dans quelle proportion par rapport aux autres éléments du dispositif.

Figure 3. Croisement des connaissances initiales des étudiants et de la progression de leurs apprentissages.

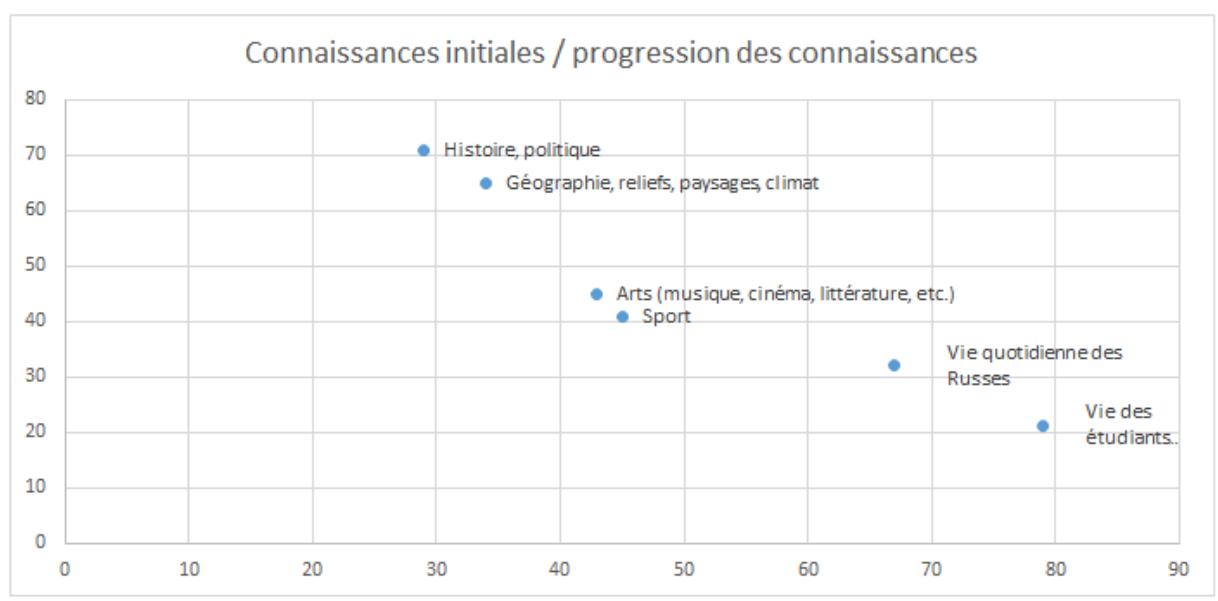


92 S'agissant des compétences interculturelles des étudiants, il s'agit, selon l'autoévaluation des étudiants, de l'écart de progression le plus important après « la vie des étudiants en Russie ", sans mention de quelle compétence précise ils développent. Il est fortement probable que les télétandems et les échanges informels que les étudiants ont mis en place de leur propre initiative aient largement contribué au développement de cette compétence. En effet, les carnets de bord des étudiants du Cnam mentionnent plus souvent les tandems pour le volet apprentissage interculturels « On [avec mon tandem] a repris les mots de vocabulaire des deux premières fiches [...], on a parlé des diminutifs des noms en russe, de la triche aux examens, des conditions d'études, des différences entre le système éducatif français et russe puis on a fini par parler de la St Valentin ».

La page Facebook est l'unique espace collectif du groupe d'étudiants du Cnam et d'Oulianovsk. Peu de signes nous permettent de voir dans cet espace une " communauté apprenante ", le terme de communauté étant un label de qualité relatif au fonctionnement des groupes et en particulier à l'intensité des interactions entre les membres (Dillenbourg, Poirier et Carles, 2003). L'espace est néanmoins convivial, il permet de choisir son télétandem, d'y puiser des éléments de conversation pour les télétandems et contribue, conjointement aux autres éléments du dispositif, au développement d'un système de références culturelles de base pour permettre l'interprétation de faits culturels et la communication interculturelle.

\section{Discussion et conclusion}

Nous dégageons à présent quelques points saillants concernant les apprentissages permis et le caractère innovant du dispositif.

S'agissant des compétences interculturelles, le projet contribue à développer un socle de connaissances culturelles et, par l'activité elle-même de sélection d'une production culturelle à partager au groupe, à prendre conscience des possibilités infinies qui s'offrent aux étudiants, à s'interroger sommairement sur le concept de culture. Or les connaissances culturelles, en tant que construction d'un réseau de références, de sens et de valeur de la culture étrangère, restent trop souvent lacunaires (Anquetil, 2006). Elles sont pourtant centrales pour interpréter des faits dans la communication interculturelle, pour mobiliser ses connaissances de façon à adopter un comportement à l'interlocuteur. Dans les retours des étudiants, nous notons toutefois de rares prises de conscience du caractère métissé des cultures, de leur caractère mouvant. Cette dimension est discutée lors de l'atelier réflexif qui clôture la formation, mais seuls les étudiants du Cnam y participent. Il s'agit d'une première limite au projet sur laquelle nous revenons plus loin.

Le choix d'une production culturelle et les commentaires qui suivent permettent la mise en œuvre de savoir-faire utiles pour la communication interculturelle, en premier lieu savoir expliciter et décomposer des pratiques culturelles sans les imposer comme des évidences. Les demandes d'informations pour interpréter plus finement un fait culturel, les comparaisons proposées, l'utilisation de différentes stratégies de séduction sociale, la mise en place de connivences culturelles sont d'autres savoir-faire utiles à la communication interculturelle. Il y a donc mobilisation et développement de certaines ressources internes et externes pour agir de façon compétente (Poumay et al., 2017) 
dans l'environnement multiculturel mais il n'y a pas lieu de parler de développement de compétences interculturelles.

D'autre part, le fait qu'aucun sujet potentiellement conflictuel n'ait été développé, qu'aucun étudiant n'ait été choqué par une publication ou des commentaires, le caractère un peu convenu des échanges, peuvent donner l'impression d'un espace sensiblement trop interculturaly correct (Ogay, 2000), un interculturel de convivialité plutôt qu'un interculturel de conflictualité. L'interculturel de convivialité cherche à aplanir les obstacles de communication plutôt qu'à les comprendre. Or, il n'est pas suffisant de chercher à aplanir les différends et éviter les conflits. Il est préférable de les vivre et de comprendre à quelles sources ils s'alimentent (Lipianski, 1999). Si le projet actuel invite, dans la séance collective de clôture avec les étudiants du Cnam, à questionner certaines zones de friction potentielles, cela n'est pas fait collectivement avec l'ensemble des étudiants, ce qui n'invite pas à une compréhension commune qui conduirait à défendre et expliquer son point de vue et ses valeurs. Il s'agit d'une deuxième limite de ce projet.

Il est aussi possible que la technologie et le réseau social Facebook invitent à un survol assez superficiel de la diversité au lieu d'une exploration profonde de la différence. C'est d'ailleurs ce que relève Kramsh (2014): «The very technology that promised to give all learners access to any foreign culture and its members is exacting its own price: shallow surfing of diversity instead of deep exploration of difference, [...] amazing ability to multitask but limited ability to problematize the task and question the question »(p.46) [La technologie qui promettait à tous les apprenants l'accès à n'importe quelle culture étrangère et à ses membres a un coût non négligeable : navigation superficielle sur la diversité au lieu d'une exploration approfondie de la différence, [...] capacité étonnante à effectuer plusieurs tâches à la fois, mais capacité limitée à problématiser la tâche et à questionner la question].

S'agissant du caractère innovant du dispositif, il se traduit par les libertés de choix offertes aux étudiants et par les responsabilités à endosser, comme organiser son apprentissage en autodirection, ses télétandems, etc. S'ajoute un accompagnement collectif et personnalisé des étudiants pour soutenir l'apprentissage. La création d'un réseau d'apprenants de deux pays via les télécollaborations pour progresser collectivement dans les apprentissages est une autre illustration de ce que peut être un projet innovant selon Fabre (2015). S'agissant de l'efficacité du projet de télécollaboration, autre composante de l'innovation pédagogique (Tricot, 2017), ses effets sont de divers ordres : il permet de mettre en réseau l'ensemble des participants en France et en Russie, de choisir un partenaire de télétandem, de développer des connaissances culturelles, de vivre des expériences interculturelles et de mettre en œuvre des savoir-faire utiles à la communication interculturelle. Cependant, de façon à mieux identifier les sujets potentiels de discorde et comprendre leurs origines, une séance collective avec l'ensemble des participants en France et en Russie, guidée par les enseignantes russes et françaises, serait susceptible d'enrichir le projet. 


\section{BIBLIOGRAPHY}

Anquetil, M. (2006). Mobilité Erasmus et communication interculturelle. Une recherche-action pour un parcours de formation. Peter Lang.

Audras, I. et Chanier, T. (2007). Acquisition de compétences interculturelles : interactions orales et écrites en tridem en ligne, Lidil, 36, 23-41. https://doi.org/10.4000/lidil.2383

Bardin, L. (2013). L'analyse de contenu ( $2^{e}$ éd., quadrige). PUF

Byram, M. (1997). Teachnig and assessing intercultural communicative competence. Multilingual Matters.

Byram, M., Zarate, G. et Neuner, G. (1997). La compétence socio-culturelle dans l'apprentissage et l'enseignement des langues. Edition du Conseil de l'Europe.

Byram, M. (2012). Reflecting on teaching 'culture' in foreign language education. Dans D., Newby (dir.), Insights into the European Portfolio for Student Teachers of Languages (EPOSTL). Cambridge Scholars Publishing.

Blattner, G. et Lomicka, L. (2012). Facebook-ing and the Social Generation: A New Era of Language Learning. Alsic, 15(1). https://doi.org/10.4000/alsic.2413

Blin, F. (2012). Introducing cultural historical activity theory for researching CMC in foreign language education. Dans M. Dooly et R. O'Dowd (dir.), Theories, Methods and Challenges. Telecollaboration in Education (p. 79-106). Peter Lang.

Chachkine, E. (2019). Tournant social des dispositifs d'apprentissage des langues en autoformation - Un exemple de dispositif pour le russe. Alsic, 22(1). https://doi.org/10.4000/ alsic.4110

Cros, F. (2014). L'innovation en formation. Dans A. Jorro (dir.), Dictionnaire des concepts de la professionnalisation (p. 169-175). De Boeck.

Culioli, A. (1990). Pour une linguistique de l'énonciation : opérations et représentations (tome 1). Ophrys. Dervin, F. (2011). Imposture interculturelle. L'Harmattan.

Dillenbourg, P., Poirier, C. et Carles, L. (2003). Communautés virtuelles d'apprentissage e.jargon ou nouveau paradigme? Dans A. Taurisson et A. et A. Sentini (dir.), Pédagogies.Net. L'essor des communautés virtuelles d'apprentissage (p. 11-47). Presses Universitaires du Québec.

Dooly, M. et O'Dowd, R. (dir.) (2012). Researching Online Foreign Language Interaction and Exchange: Theories, Methods and Challenges. Peter Lang. https://doi.org/

10.3726/978-3-0351-0414-1

Fabre, M. (2015). L'innovation pédagogique. Journées d'études du 25 juin 2015 de l'université de Nantes. L'innovation pédagogique. Pourquoi? Pour qui? [vidéo]. Université de Nantes. https://webtv.univnantes.fr/fiche/6678/michelfabre-l-innovation-pedagogique-pourquoi-pour-qui

Galisson, R. (2000). La pragmatique lexiculturelle pour accéder autrement, à une autre culture, par un autre lexique. Mélanges CRAPEL, 25, 47-73.

Kramsch, C. (2014). Language and culture. AILA Review, 27(1), 30-55.

Lewis, T. et O'Dowd, R. (dir.) (2016). Online Intercultural Exchange: Policy, Pedagogy, Practice. Routledge. 
Lewis, T., O'Rourke, B. et Dooly, M. (dir.) (2016). Online Intercultural Exchange: Innovation in Language Learning and Teaching, 10(1). 10.1080/17501229.2015.1133541

Lomicka, L. et Lord, G. (2016). Social networking in language learning. Dans F. Farr, L. Murray (dir.), The Routledge Handbook of Language Learning and Technology (p. 255-268). Routledge.

Muller, C. (2015). Des échanges interculturels à distance : oui, mais quelle vision de l'interculturel? Dans A. Potolia et D. Lemay, D. (dir.), Enseignement/apprentissage des langues et pratiques numériques émergentes (p. 113-125). Éditions des archives contemporaines.

Lipianski, E. M. (1999). Perception de l'autre et pédagogie des rencontres. Dans J. Demogon et E. D. Lipianski (dir.), Guide de l'interculturel en formation (p. 81-87). Retz.

O'Dowd, R. (2006). Combining networked communication tools for students' ethnographic research. Dans Belz, J. A. et Thorne, S. L. (dir.), Internet mediated intercultral foreign language education (p. 147-176). Thomsen et Heinle.

O’Dowd, R. et Ritter, M. (2006). Understanding and working with "failed communication" in tellecollaborative exchange. Calico Journal, 23(3). 623-642. https://calico.org/html/article_112.pdf

Ogay, T. (2000). De la compétence à la dynamique interculturelle. Peterlang.

Poumay, M., Tardif, J. et Georges, F. (éd.). (2017). Organiser la formation à partir des compétences : Un pari gagnant pour l'apprentissage dans le supérieur. De Boeck Supérieur.

Tricot, A. (2017). L'innovation pédagogique. Mythes et réalités. Retz.

UNESCO. (2013). Compétences interculturelles. Cadre conceptuel et opérationnel. Organisation des Nations Unis pour l'éducation, la science et la culture.

Vygotski, L. S. (1934 / 1997). Pensée et langage. La Dispute.

Zarate, G. (1986). Enseigner une culture étrangère. Hachette.

\section{ABSTRACTS}

This article deals with a project of cultural exchange on a Facebook page between students of the Cnam (France) and students of the University of Ulyanovsk (Russia). The project of (inter)cultural telecollaboration is grounded in Vygotski's (1934/1997) socio-cultural theory. First, an analysis of the types of knowledge exchanged between participants as well as the skills mobilised for intercultural communication during the discussions is carried out. To achieve this, the typology of socio-cultural competences for intercultural communication developed by Anquetil (2006) is adopted. Then, the innovative character of the project is examined, relating it to the main academic pedagogical innovations of the moment identified by Fabre (2015), and its effectiveness (Tricot, 2017), another purpose of pedagogical innovation, is considered.

Cet article porte sur un projet d'échanges de productions culturelles sur une page Facebook entre étudiants du Cnam (France) et étudiants de l'université d'Oulianovsk (Russie). Ce projet de télécollaboration (inter)culturelle s'ancre dans la théorie socioculturelle de Vygotski (1934/1997). Dans un premier temps, nous analysons les types de savoirs échangés entre participants ainsi que les savoir-faire mobilisés pour la communication interculturelle lors des échanges. Pour cela, nous adoptons la typologie des compétences socioculturelles pour la communication interculturelle élaborée par Anquetil (2006). Ensuite, nous nous interrogeons sur le caractère innovant du projet, en le rapportant aux principales innovations pédagogiques universitaires du 
moment identifiées par Fabre (2015), et sur une autre finalité de l'innovation pédagogique, l'efficacité (Tricot, 2017).

INDEX

Mots-clés: télécollaboration (inter)culturelle; savoirs culturels; savoir-faire pour la communication interculturelle; innovation pédagogique

\section{AUTHOR}

\section{ELSA CHACHKINE}

Conservatoire national des arts et métiers, Paris, France, elsa.chachkine@lecnam.net 